\title{
Negative First-Order Quasi-Phase Matching
}

\author{
Yannick Petit, ${ }^{1,2}$ Alexandra Peña, ${ }^{3}$ Patricia Segonds, ${ }^{3}$ Jérôme Debray, ${ }^{3}$ Simon Joly, ${ }^{4}$ Andrius Zukauskas, ${ }^{5}$ \\ Fredrik Laurell, ${ }^{5}$ Valdas Pasiskevicius, ${ }^{5}$ Carlota Canalias, ${ }^{5}$ and Benoît Boulanger ${ }^{3, *}$ \\ ${ }^{1}$ Université de Bordeaux, CNRS, CEA, CELIA, UMR 5107, F-33405 Talence, France \\ 2Université de Bordeaux, CNRS, ICMCB, UPR 9048, F-33608 Pessac, France \\ ${ }^{3}$ Université Grenoble Alpes, CNRS, Grenoble INP, Institut Néel, 38000 Grenoble, France \\ ${ }^{4}$ IMS Laboratory, University of Bordeaux, UMR CNRS 5218, 351 Cours de la Libération, 33405 Talence, France \\ ${ }^{5}$ Laser Physics, Applied Physics Department, Royal Institut of Technology, AlbaNove University Center, Roslagstullsbacken 21, 10691 \\ Stockholm, Sweden \\ benoit.boulanger@neel.cnrs.fr
}

\begin{abstract}
We propose a new scheme of quasi-phase matching based on the negative first-order of the spatial modulation of the sign of the second-order optical nonlinearity. The experimental validation is done in a periodically-poled Rubidium-doped $\mathrm{KTiOPO}_{4}$ crystal. (c) 2020 The Author(s)

OCIS codes: (160.4330) Nonlinear optical materials; (190.2620) Harmonic generation and mixing.
\end{abstract}

\section{Introduction}

Phase-matching is the privilege way to optimize the performance of nonlinear optical frequency conversion. Usually, it can be obtained by using the birefringence of anisotropic crystals, which corresponds to birefringence phasematching (BPM). But an alternative is quasi-phase matching (QPM), that is based on a periodical modulation of the sign of the second-order optical nonlinear coefficient of anisotropic or isotropic crystals. We propose here a new scheme of QPM that allows us to enlarge both the spectral range of phase-matched generation and the spectral acceptance. We validated this scheme by the study of Second-Harmonic Generation (SHG) in the ferroelectric periodically-poled Rubidium-doped $\mathrm{KTiOPO}_{4}$ crystal (PPRKTP).

\section{Theory}

The key parameter of QPM is the periodicity $\Lambda$ of the spatial modulation of the second-order nonlinearity along the direction of propagation of three interacting waves such as their circular frequencies fulfil: $\omega_{3}-\omega_{1}-\omega_{2}=0$. Actually, the goal is to compensate the spatial phase-mismatch $\left(\overrightarrow{\Delta \mathrm{k}}=\overrightarrow{k_{3}}-\overrightarrow{k_{1}}-\overrightarrow{k_{2}}\right)$ between the induced polarization and the radiated field. Considering the usual collinear propagation scheme, the QPM relation writes as the following scalar equality [1]:

$$
\Lambda=m \frac{2 \pi}{\Delta k}
$$

where $m$ is the order of the Fourier series. Usually, $m$ is chosen as a positive integer ( $m \geq 1$ ), so that the phase-mismatch of the direction that is considered has to be a positive quantity $(\Delta \mathrm{k}>0)$. It is shown in Fig. 1 (left) in the optimal case, i.e. $m=1$ that is the classical scheme of QPM that we called QPM-A.

But it is quite possible to also consider directions of propagation for which $\Delta \mathrm{k}<0$, which has never been reported before to the best of our knowledge. In that case, Eq. (1) is fulfilled for $m \leq-1$. We called this new scheme QPM-B. As for the case of positive orders, the lowest QPM-B order (namely $m=-1$ ) leads to the best efficiency compared to higher QPM-B orders (namely $\mathrm{m}<-1$ ), which requires the shortest needed QPM periodicity, as shown in Fig. 1 (right).

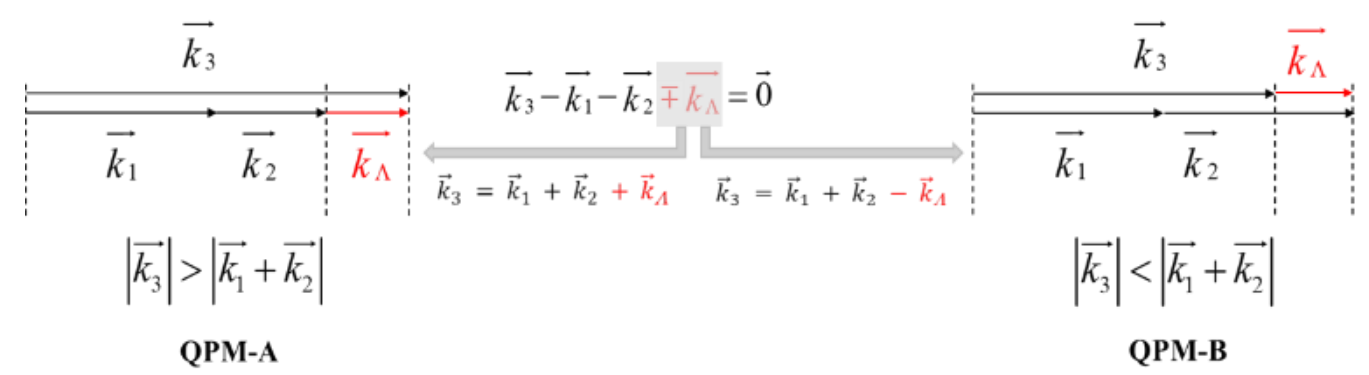

Fig.1 Schemes of collinear Quasi-Phase-Matching (QPM). $\overrightarrow{k_{\Lambda}}$, with $\left|\overrightarrow{k_{\Lambda}}\right|=\frac{2 \pi}{\Lambda}$, is the grating vector along the propagation direction where $\Lambda$ is the periodicity of the spatial modulation of the sign of the second-order non-linearity; $\overrightarrow{k_{1}}, \overrightarrow{k_{2}}$ and $\overrightarrow{k_{3}}$ are the wave vectors of the interacting waves: positive-order QPM-A (left), negative-order QPM-B (right). 
New efficient directions for parametric frequency conversion are expected using QPM-B since the corresponding phase-matching relation is different from that of QPM-A.

\section{QPM measurements}

We validated this theory by an experiment of SHG $(\omega+\omega \rightarrow 2 \omega)$ performed in a PPRKTP with a grating period $\Lambda=$ $38.52 \mu \mathrm{m}$. We previously used this crystal to demonstrate the existence of angular-quasi-phase-matching (AQPM) in the case of the classical QPM-A, as shown in Fig. 2 [2]. AQPM, both with schemes A or B, is an interesting configuration because it allows the waves to propagate at any angle with respect to the grating vector $\overrightarrow{k_{\Lambda}}$, then giving access to new types of QPM in new spectral ranges and giant spectral acceptances.

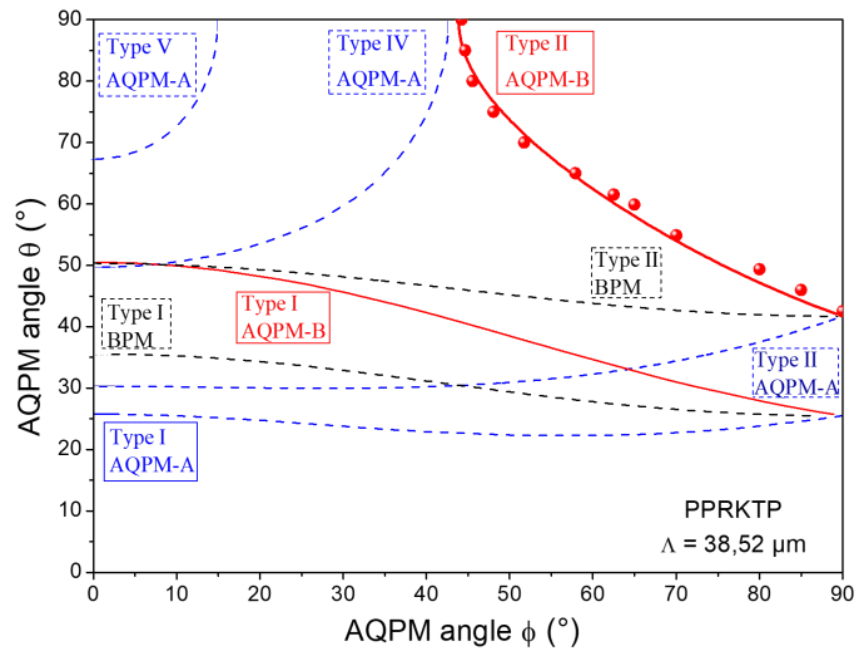

Fig. 2. Second-Harmonic Generation corresponding to a fundamental wavelength $\lambda_{\omega}=2.15 \mu \mathrm{m}$ in a PPRKTP crystal with a grating period $\Lambda=$ $38.52 \mu \mathrm{m}$; Types I and II BPM (black dashed lines : calculations from [2]), Types I, II, IV and V AQPM-A (blue dashed lines : calculations from [2]), Types I and II AQPM-B (red continuous lines : calculations - red dots : experimental data [present work]). $\theta$ and $\phi$ are the angles of spherical coordinates in the dielectric frame $(\mathrm{x}, \mathrm{y}, \mathrm{z}): \phi=0^{\circ}$ corresponds to the xz plane, $\phi=90^{\circ}$ to the yz plane, and $\theta=90^{\circ}$ to the $\mathrm{xy}$ plane.

For comparison, we considered the same fundamental wavelength than that used for the study of AQPM-A, i.e. $\lambda_{\omega}$ $=2.15 \mu \mathrm{m}$. At this wavelength, AQPM-B is expected to be possible for both type I (same polarization for the two waves at $\omega$ ) and type II (orthogonal polarizations for the two waves at $\omega$ ) according to our calculations and by using the Sellmeier equations of reference [3], as shown in Fig.2.

The PPRKTP crystal was cut as a sphere, with a diameter of $4.76 \mathrm{~mm}$, in order to have a full angular tunability for the SHG experiments. The sphere is oriented by X-rays and stuck on a goniometric head placed in a Kappa circle so that it can rotate on itself. The fundamental beam is emitted by a nanosecond BBO Optical Parametric Oscillator, the wavelength being fixed at $2.15 \mu \mathrm{m}$. The conversion efficiency of Type I AQPM-B being more than one order of magnitude smaller than that of type II, as it is the case for BPM, we focused on Type II. The agreement between the measured and calculated phase-matching curves is excellent, as shown in Fig. 2.

The calculation of Type II AQPM-B at any wavelength in PPRKTP reveals that there is a giant wavelength acceptance, i.e. $\mathrm{L} . \delta \lambda=76 \mathrm{~nm} . \mathrm{cm}$, at $\lambda_{\omega}=1.567 \mu \mathrm{m}$ that is located in the telecom range.

\section{Conclusion}

We propose and validated the scheme of negative-first-order quasi-phase-matching from the study of the biaxial PPRKTP crystal. This original study extends the body of knowledge on quasi-phase matching and can of course be applied to other materials such as the uniaxial MgO:PPLN crystal.

\section{References}

[1] M. M. Fejer, G. A. Magel, D. H. Jundt, and R. L. Byer, "Quasi-phase-matched second harmonic generation: tuning and tolerances," IEEE Journal of Quantum Electronics 28, 2631-2654 (1992).

[2] D. Lu, A. Peña, P. Segonds, J. Debray, S. Joly, A. Zukauskas, F. Laurell, V. Pasiskevicius, H. Yu, H. Zhang, J. Wang, C. Canalias, B. Boulanger, "Validation of the angular quasi-phase-matching theory for the biaxial optical class using PPRKTP," Optics Letters 43, 4276-4279 (2018).

[3] K. Kato and E. Takaoka, "Sellmeier and thermo-optic dispersion formulas for KTP," Applied Optics 41, 5040-5044 (2002). 